EPJ Web of Conferences 59, 05001 (2013)

DOI: $10.1051 /$ epjconf/20135905001

(C) Owned by the authors, published by EDP Sciences, 2013

\title{
Comparison of different approaches to the numerical calculation of the LMJ focal
}

\author{
A. Bourgeade, H. Coïc and J.-P. Goossens
}

CEA, DAM, CESTA, BP. №2, 33114 Le Barp, France

\begin{abstract}
The beam smoothing in the focal plane of high power lasers is of particular importance to laserplasma interaction studies in order to minimize plasma parametric and hydrodynamic instabilities on the target. Here we investigate the focal spot structure in different geometrical configurations where standard paraxial hypotheses are no longer verified. We present numerical studies in the cases of single flat top square beam, LMJ quadruplet and complete ring of quads with large azimuth angle. Different calculations are made with Fresnel diffraction propagation model in the paraxial approximation and full vector Maxwell's equations. The first model is based on Fourier transform from near to far field method. The second model uses first spherical wave decomposition in plane waves with Fourier transform and propagates them to the focal spot. These two different approaches are compared with Miró [1] modeling results using paraxial or Feit and Fleck options. The methods presented here are generic for focal spot calculations. They can be used for other complex geometric configurations and various smoothing techniques. The results will be used as boundary conditions in plasma interaction computations.
\end{abstract}

\section{PARAXIAL AND NON PARAXIAL MODELING}

\subsection{Theoretical approach}

A realistic description of the laser beam is necessary as boundary condition for NIF and LMJ plasma calculus [2]. Ray tracing is commonly used to define such boundary conditions [3]. We present here a different approach based on plane wave decomposition. For paraxial approximation, the field expression after the focusing lens (focal length $z_{f}$ ) is given by parabolic approximation of the spherical phase of the lens:

$$
F(x, y)=f(x, y) \exp \left[-i \frac{k_{0}}{2 z_{f}}\left(x^{2}+y^{2}\right)\right]
$$

where $f(x, y)$ is the amplitude of the field with a spatial extension size of $a$ and $k_{0}=\omega / c$ is the wave number of the field. The field expression in the $k$ space (spatial frequencies) with one fast Fourier transform (FFT) requires a large number of points. But for a parabolic phase lens, plane wave decomposition of the electric field in the $k$ space with double FFT allow to reduce the spatial sampling (2048 points instead of 256000 for $\mathrm{a}=1.2 \mathrm{~m}$ and $z_{f}=0.8 \mathrm{~m}$ ). The plane wave decomposition with two FFT is given by:

$$
\begin{gathered}
\hat{F}\left(k_{x}, k_{y}\right)=-i \frac{z_{f}}{k_{0}} \exp \left[i \frac{z_{f}}{2 k_{0}}\left(k_{x}^{2}+k_{y}^{2}\right)\right] F F T_{u, v}\left\{\exp \left[-i \frac{z_{f}}{2 k_{0}}\left(u^{2}+v^{2}\right)\right]\right. \\
\left.F F T_{x, y}\{f(x, y)\}\right\}\left(-\frac{z_{f} k_{x}}{k_{0}},-\frac{z_{f} k_{y}}{k_{0}}\right) .
\end{gathered}
$$

This is an Open Access article distributed under the terms of the Creative Commons Attribution License 2.0, which permits unrestricted use, distribution, and reproduction in any medium, provided the original work is properly cited. 

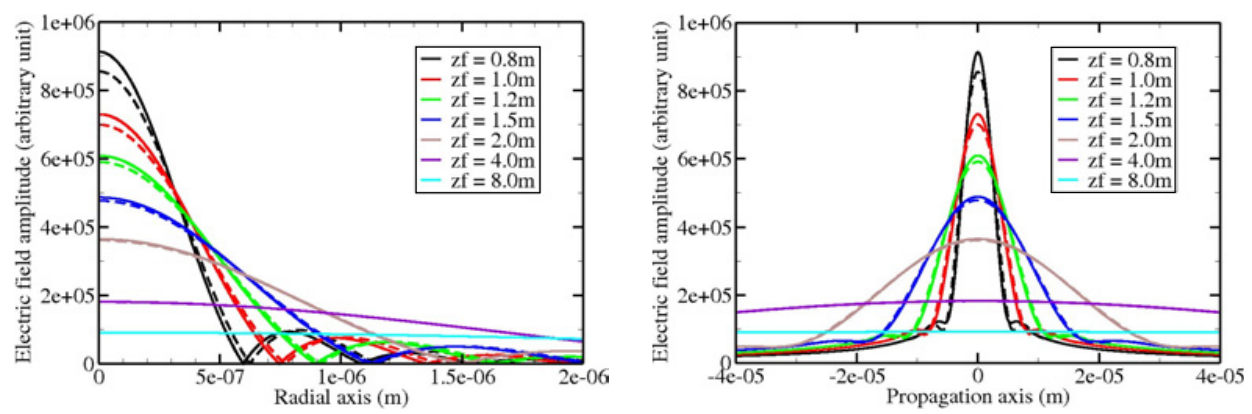

Figure 1. Electric field distribution along radial axis (a) and along propagation axis (b) for different focal lengths. Solid lines represent the paraxial model and dashed lines the non paraxial model.

A more complete analysis of this approach can be found in reference [4]. We consider, for non paraxial calculus, only the difference between spherical and parabolic phase lens. Then, the field expression after the focusing lens can be written as:

$$
F(x, y)=f(x, y) \exp \left[-i k_{0}\left(\sqrt{x^{2}+y^{2}+z_{f}^{2}}-z_{f}\right)\right]=g(x, y) \exp \left[-i \frac{k_{0}}{2 z}\left(x^{2}+y^{2}\right)\right] .
$$

Then, non paraxial $k$ space distribution is similar to the previous expression by replacing $f(x, y)$ by $g(x, y)$. The non paraxial term propagation [5] is giving a general expression for the field. The recombination of the waves is obtained without FFT but with standard Fourier transform and then no step by step propagation is needed:

$$
E(x, y, z)=\frac{1}{2 \pi} \iint \hat{F}\left(k_{x}, k_{y}\right) \exp \left\{i .\left[x . k_{x}+y \cdot k_{y}+z \cdot\left(\sqrt{k_{0}^{2}-k_{x}^{2}-k_{y}^{2}}-k_{0}\right)\right]\right\} d k_{x} d k_{y} .
$$

\subsection{Numerical codes}

Maxwell modeling is obtained with a specific code [4], including paraxial and Maxwell (non parabolic) as well as asymptotic approaches with field summation. On the other hand, the Miró code can deal with both parabolic and non parabolic modeling. In such a case, the calculi are performed with adaptative Fresnel diffraction mode (afd), i.e. only the differences between parabolic phases are propagated. For parabolic calculus, a complete calculus requires only a few points, even for large apertures. But for the non paraxial case, and for large aperture, the difference between parabolic and non parabolic phase become large and the spatial frequency domain goes up to $6.10^{5} \mathrm{~m}^{-1}$ and then a complete calculus requires a lot of points.

Figure 1(a) represents the electric field distribution along the radial axis with paraxial and non paraxial approximations for different focal lengths. The maximum electric field peak is reduced by $7 \%$ for $z_{f} / a=1,6\left(z_{f}=0.8 \mathrm{~m}\right)$. Fig. 1(b) represents the electric field distribution along the $z$ axis with paraxial and non paraxial approximations for different focal lengths. After the first rebound, the electric field is decreasing and paraxial and Maxwell approaches give similar results.

\section{LMJ FOCAL SPOT WITHOUT BEAM SMOOTHING TECHNICS}

\subsection{Focal spot structure for paraxial and non paraxial modeling}

LMJ Quadruplets are composed of four $356 \mathrm{~mm} \times 370 \mathrm{~mm}$ single beams at $\lambda=351 \mathrm{~nm}$ focused with $8 \mathrm{~m}$ focusing gratings (each quad has its own gratings). The cone is built with amplitude and phase summation of five elementary quads projected in the $(x, y)$ plane with common $k_{z}$ propagation 


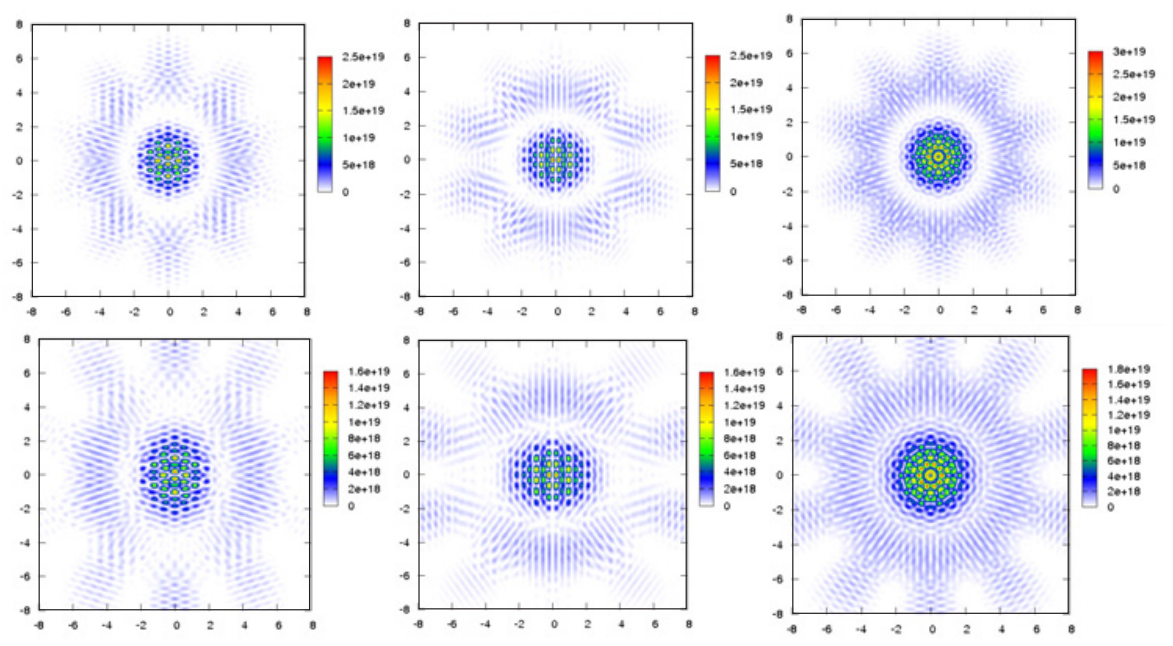

Figure 2. From left to right: $\left|E_{x}\right|^{2},\left|E_{y}\right|^{2}$ and total intensity $\left|E_{x}\right|^{2}+\left|E_{y}\right|^{2}$ for paraxial calculus (upper) and non paraxial calculus (lower) with cone projected in a plan.

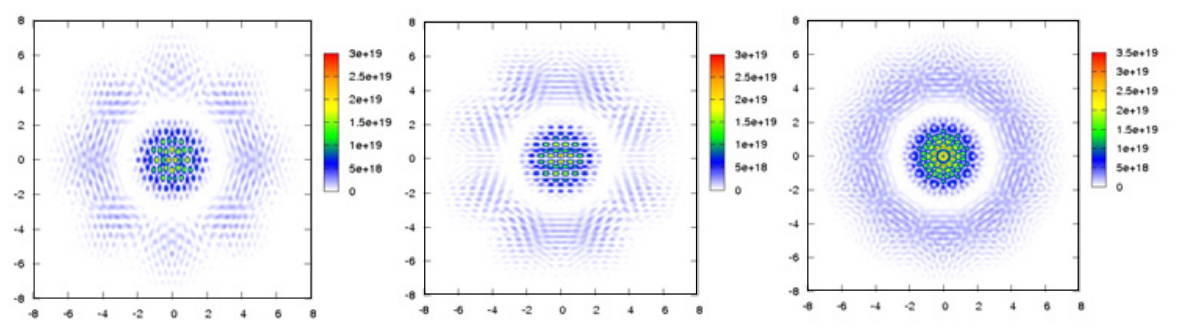

Figure 3. From left to right: $\left|E_{x}\right|^{2},\left|E_{y}\right|^{2}$ and total intensity $\left|E_{x}\right|^{2}+\left|E_{y}\right|^{2}$ for Maxwell calculus. Each quad is treated with individual $k_{z}$ component.

direction (fig. 2.). The resulting intensity map is focused with $8 \mathrm{~m}$ equivalent lens, which is equivalent to operate Fourier transformation of the field. Intensity profiles $\left|E_{x}\right|^{2}$ and $\left|E_{y}\right|^{2}$ are not symmetric because of non symmetric $(x, y)$ polarisation basis, while $\left|E_{x}\right|^{2}+\left|E_{y}\right|^{2}$ recovers symmetric structure. The 2D non paraxial calculus for a complete cone $\left(z_{f} / a=1.6\right)$ requires at least $16384 \times 16384$ points, and the computer memory limit is actually $8192 \times 8192$ points. Thus asymptotic expansion is used for the Fourier transform.

\subsection{Focal spot structure with Maxwell modeling}

With Maxwell modeling, the focal spot is obtained with an independant summation of elementary quads with different $k_{z}$ propagation direction. Maxwell modeling is calculating $\left|E_{x}\right|^{2},\left|E_{y}\right|^{2}$ and $\left|E_{z}\right|^{2}$ distribution of the total field while the Miró code is not considering $\left|E_{z}\right|^{2}$ distribution.

Fig. 3. represents the intensity maps obtained with Maxwell calculus $(2048 \times 2048$ points for each quad). In our case, the $E_{z}$ component of the field is lower than $E_{x}, E_{y}$ components: $\left|E_{z}\right|^{2} \approx 10^{-3}\left|E_{x, y}\right|^{2}$. Surprisingly, the intensity maps from summation of individual quads are closer to the paraxial calculus with a cone projected in a plane than a non paraxial one with the same incident field (Fig. 2.). Thus, such a paraxial calculus is a good approximation for large $z_{f} / a$ ratio (up to 1.6). 

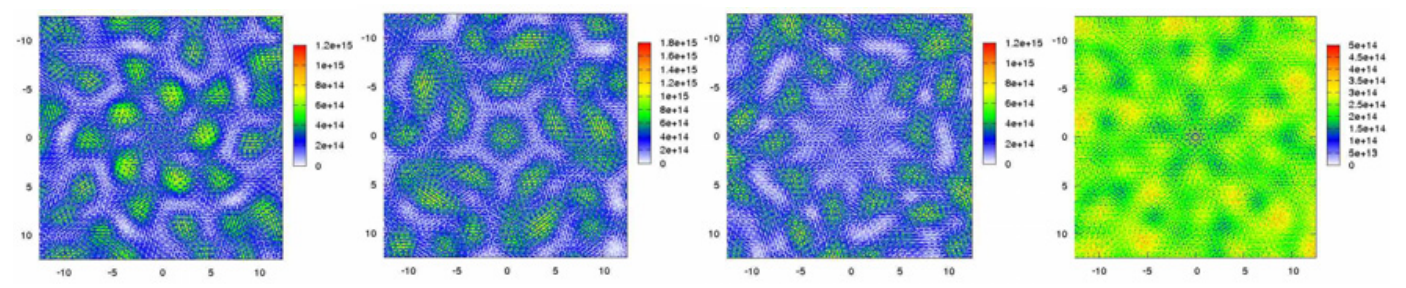

Figure 4. Smoothed focal spot in $X Y$ plan for (a) $\mathrm{m}=-9$, (b) $\mathrm{m}=0$, (d) $\mathrm{m}=+9$ spectrum Bessel lines and (c) summation of all spectral components of the field.

\section{SMOOTHED FOCAL SPOT}

The smoothed focal spot is obtained by inserting a phase plate before the lens for each beam (spatial smoothing). Temporal smoothing is obtained with a temporal phase modulation of the beam (depth of $m \mathrm{rad}$ ) which is angularly dispersed in time with the focusing grating and produces $2 m$ Bessel lines in spectral domain.

Fig. 4. represents the smoothed focal spot for $33.2^{\circ}$ cone in the $X Y$ plan $(25 \mu \mathrm{m}$ scale in $X$ and $Y)$ for different spectral lines and the final integrated smoothed pattern over the overall spectral components of the field. Let us notice a reduction of the hot spots by a factor about 3 .

\section{CONCLUSION}

A full vector Maxwell model is considering $E_{x}, E_{y}$ and $E_{z}$ electric field components while paraxial and non paraxial models (Miró software) are considering only $E_{x}$ and $E_{y}$ components. Plasma is sensible to the direction of the electric field and to its instantaneous value. And because of non linear effects, the way the fields are summed can be crucial, it is not only a question of energy. Paraxial approximation is still valid for low $z_{f} / a$ aperture ratio because of both approximation on lens and propagator phase expression. For beams with plane distribution, the paraxial approximation is still valid until $z_{f} / a \approx 5$, ( $<1 \%$ error). For LMJ cone distribution (spherical distribution) the paraxial approximation is still valid until $z_{f} / a \approx 1.6\left(33.2^{\circ}\right.$ cone $)$.

\section{References}

[1] O. Morice, Opt. Eng. 14, 1530-1541 (2003)

[2] L. Divol et al., Phys. Plasmas 15, 056313 (2008)

[3] T. B. Kaiser, Phys. Rev. E, 61, 895-905 (2000)

[4] A. Bourgeade and B. Nkonga, ESAIM Proc., 32, 1-17 (2011)

[5] M. D. Feit and J. A. Fleick, Jr, J. Opt. Soc. Am. B, 5, 633-640 (1988) 